9th ICATPP Conference on Astroparticle, Particle, Space Physics, Detectors and Medical Physics Applications

\title{
Analysis of Test-Beam Data from a Prototype LHCb RICH Detector
}

Mitesh Patel, CERN
(on behalf of the LHCb RICH Group)
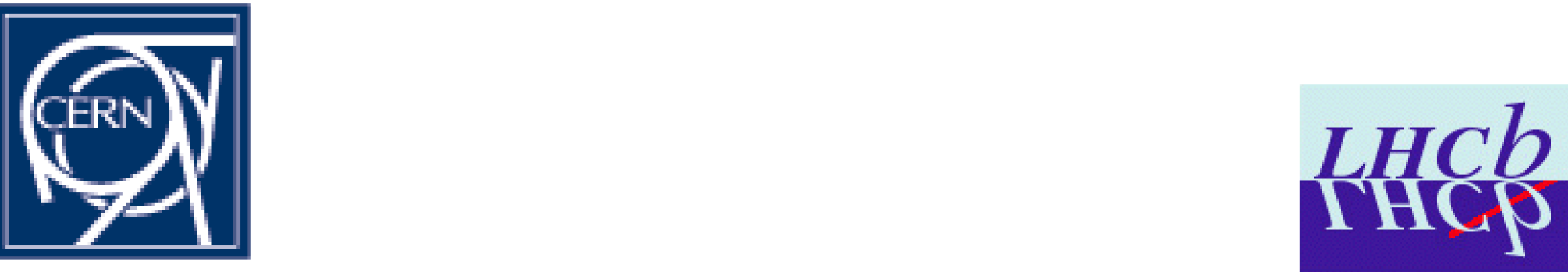


\section{LHCb Experimental Goals}

- Precision Measurements of CP Violation and rare b decays

- Large Samples of b decays at LHC

- $\mathrm{N}_{\mathrm{bb}}=10^{12}$ / year with Luminosity $210^{32} \mathrm{~cm}^{-2} \mathrm{~s}^{-1}$ for $10^{7} \mathrm{~s}$

- b production predominately at small polar angle

$\Rightarrow$ LHCb optimized as single forward arm spectrometer

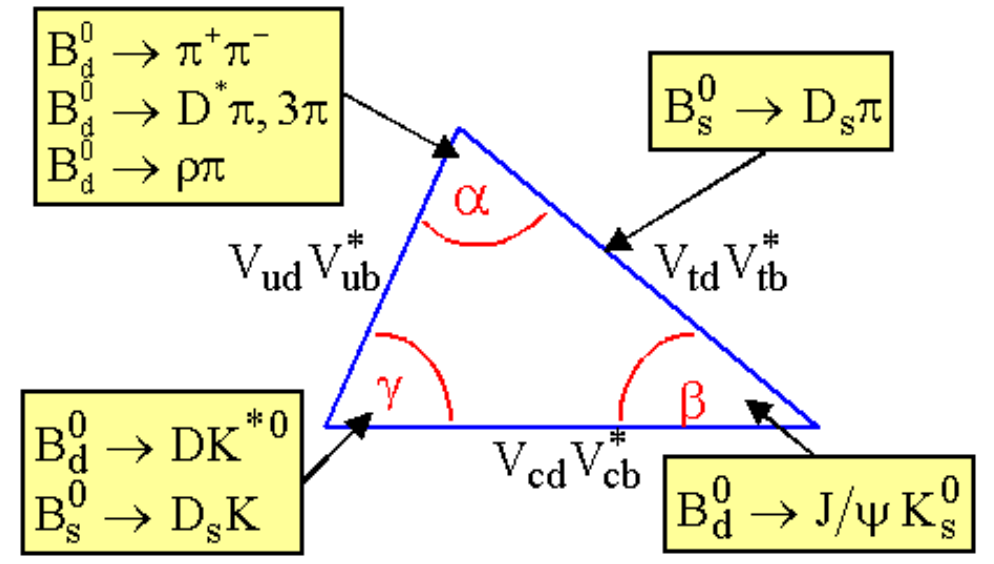

Example decays
- Many pure hadronic final states

$\Longrightarrow$ Particle identification $(\pi / K)$ essential

- Employ two RICH detectors

$\Rightarrow$ This talk: beam test of a RICH prototype

- Test of photon detector in beam conditions

- Integration of readout electronics

- Mechanics, power, cooling, insulation... 


\section{LHCb Experiment}

- Dedicated B physics Experiment at the LHC

- Acceptance :

- 15-300mrad (bending)

- 15-250mrad RICH2 (non-bending) $\quad Z \sim 9.5-11.9 \mathrm{~m}$

Muon System Z 15.0-20.0 m

- Particle ID

- RICH detectors

- Calorimeters

- Muon Detectors

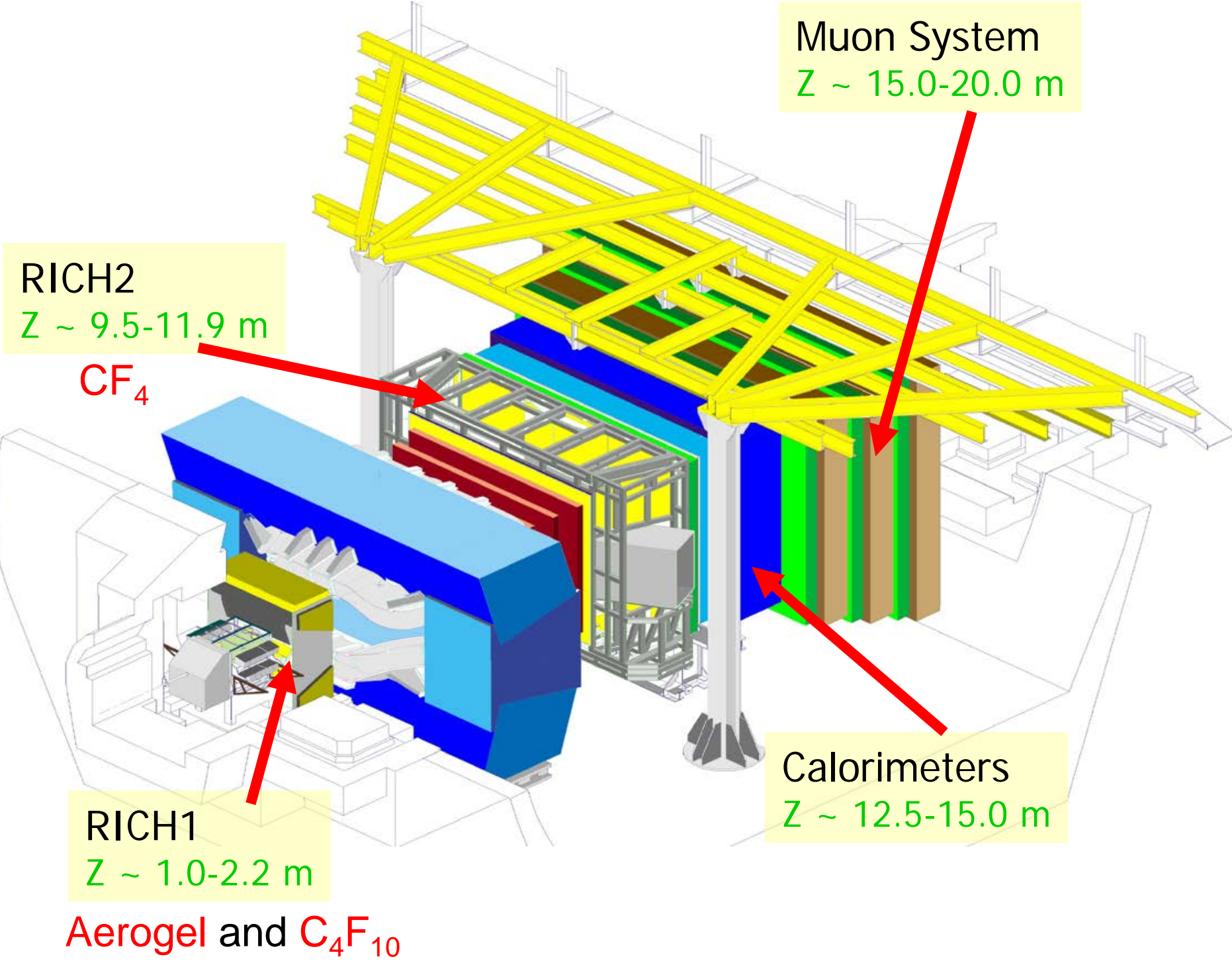




\section{RICH System Overview}
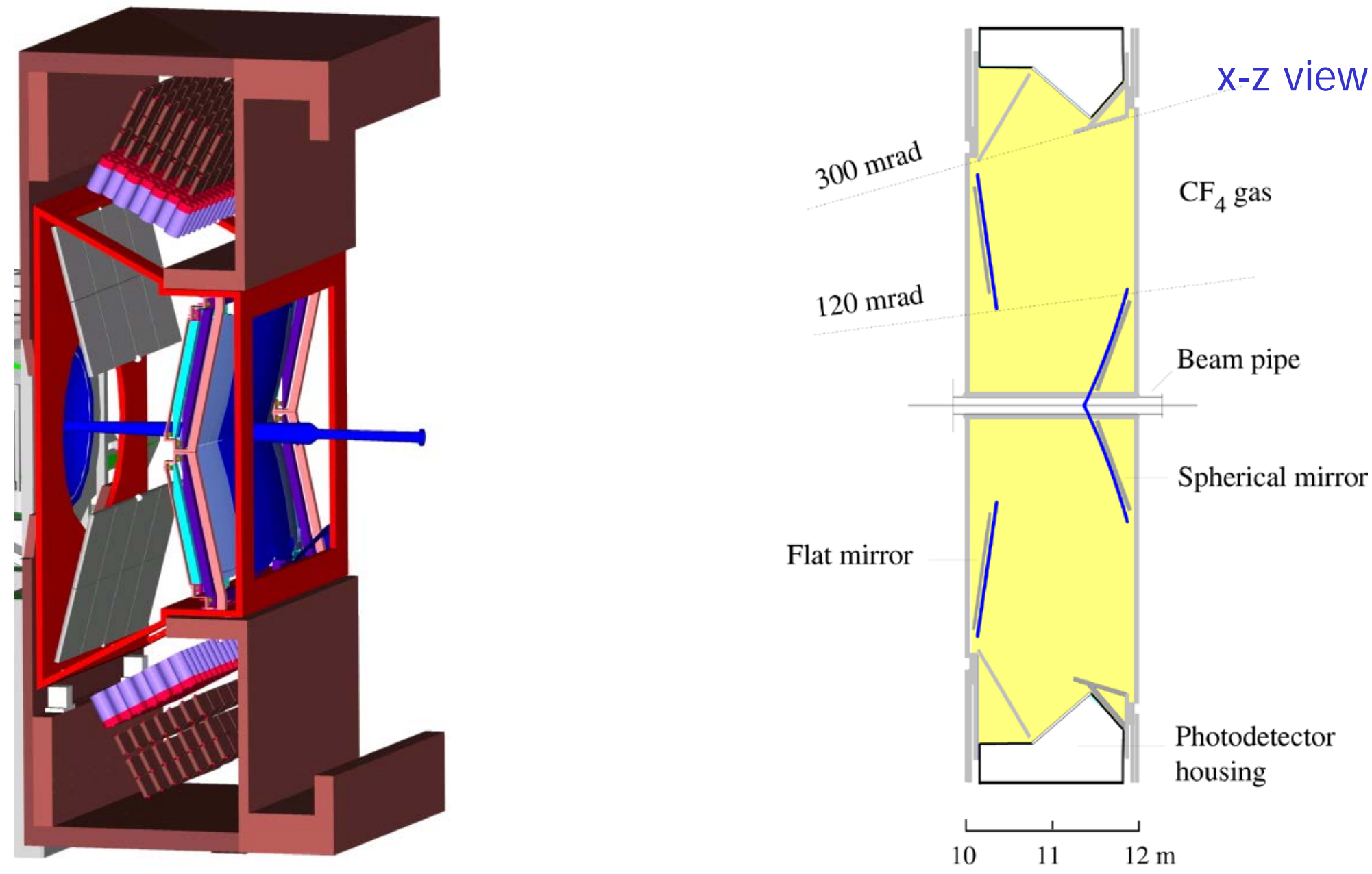

\section{RICH1:}

$5 \mathrm{~cm}$ aerogel $\mathrm{n}=1.03, \quad 2-11 \mathrm{GeV}$ $4 \mathrm{~m}^{3} \quad \mathrm{C}_{4} \mathrm{~F}_{10} \quad \mathrm{n}=1.0014, \quad 10-60 \mathrm{GeV}$

\section{$\mathrm{RICH}$ :}

$100 \mathrm{~m}^{3} \mathrm{CF}_{4} \quad \mathrm{n}=1.0005, \quad 17-100 \mathrm{GeV}$ 
- HPD developed in close collaboration with industry

- Extensive programme of lab measurements $\Rightarrow$ device is well understood, operates as expected in the lab

- Beam-tests of first six pre-production HPDs before launching production of $\sim 550$ !

- Integration Issues :

from:

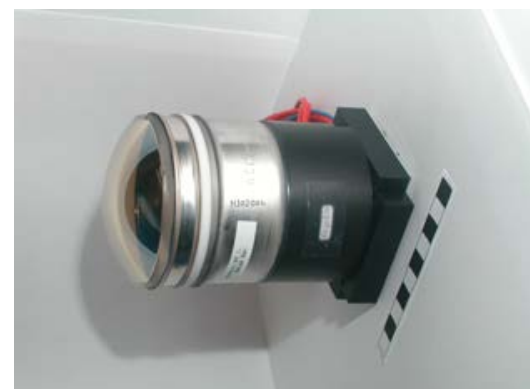

One single HPD

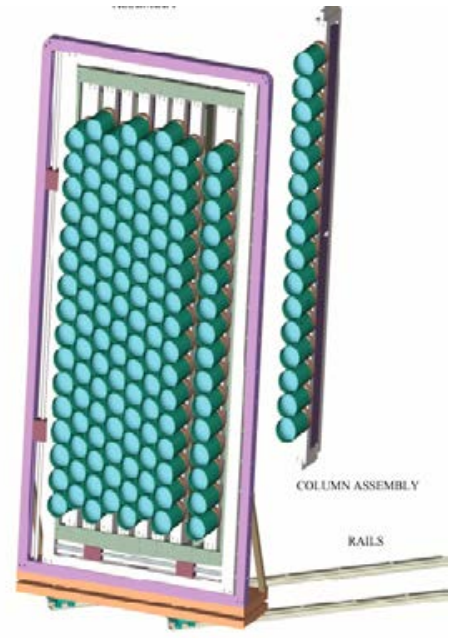

One HPD column

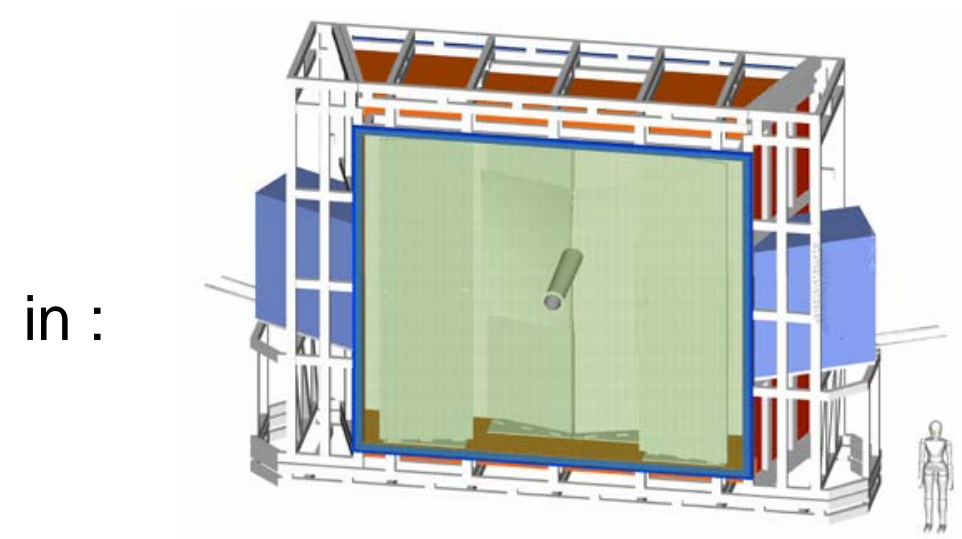

One RICH detector

- Need to ensure that the HPD and other elements of the RICH detector will work in situ i.e. test :

- Complete readout electronics chain

- Mechanics

- Power

- Cooling, insulation ....

Establish these elements work as in the lab using a standard candle 


\section{The Prototype RICH Detector}

- A prototype RICH detector was constructed
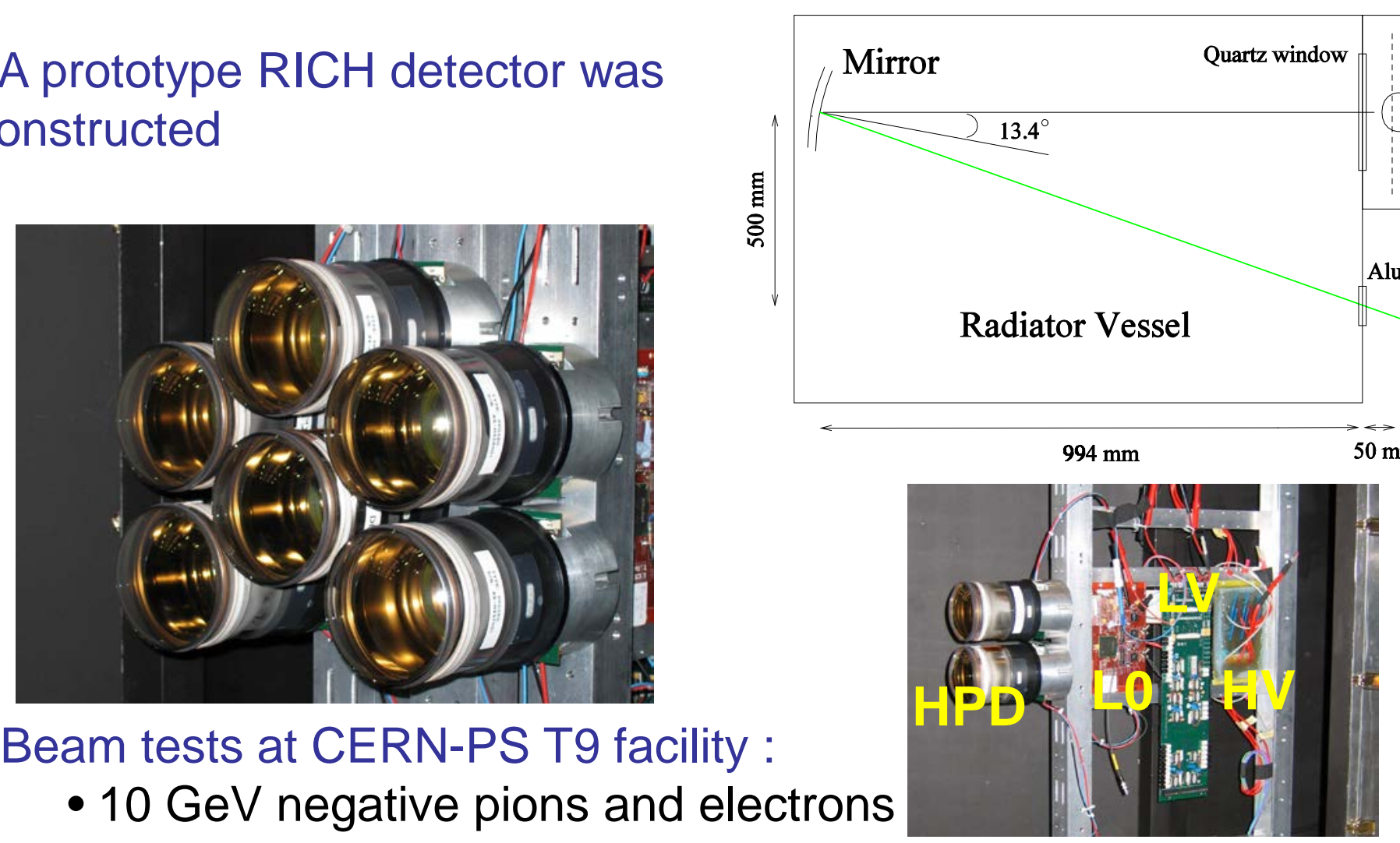

- Beam tests at CERN-PS T9 facility :

- $10 \mathrm{GeV}$ negative pions and electrons

- Equip 3 columns with first six pre-production HPDs

- $\mathrm{N}_{2}$ radiator- optics such that electron ring encompassed on single HPD

- $\mathrm{C}_{4} \mathrm{~F}_{10}$ radiator - rings cover up to four HPDs

- Measure HPD detection efficiency with prototype on- and off-detector electronics operating together in beam conditions 


\section{RICH Readout Electronics}

1. Pixel chip, encapsulated in HPD

- Binary data multiplexed out at $40 \mathrm{MHz}$

2. Level-0 Board

- Drives the pixel chip

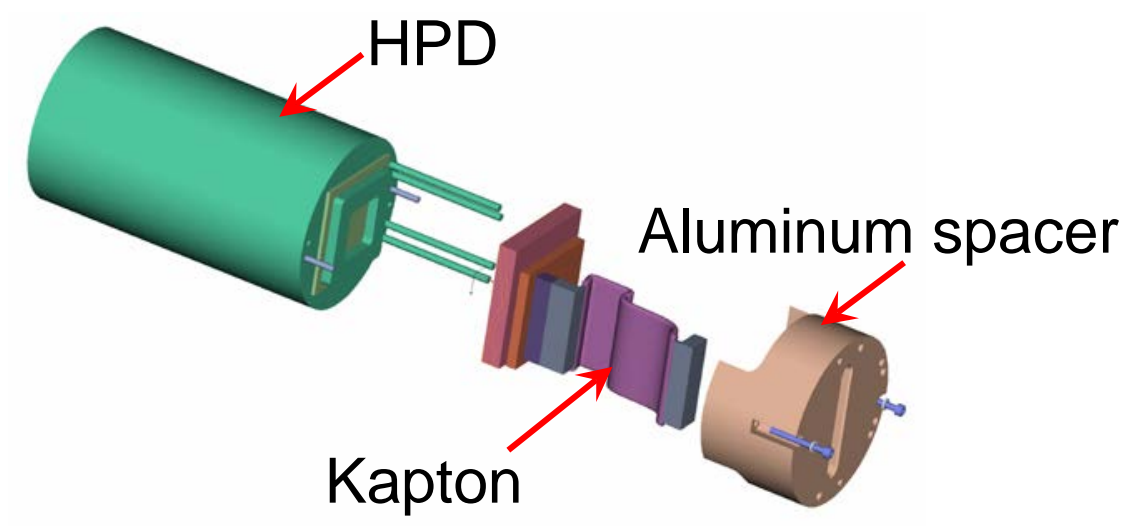

- Distributes clocks, triggers via TTC

- Controls DC power levels for pixel chip

- Gbit optical links (in LHCb 100 m to counting room)

- Controlled using JTAG interface
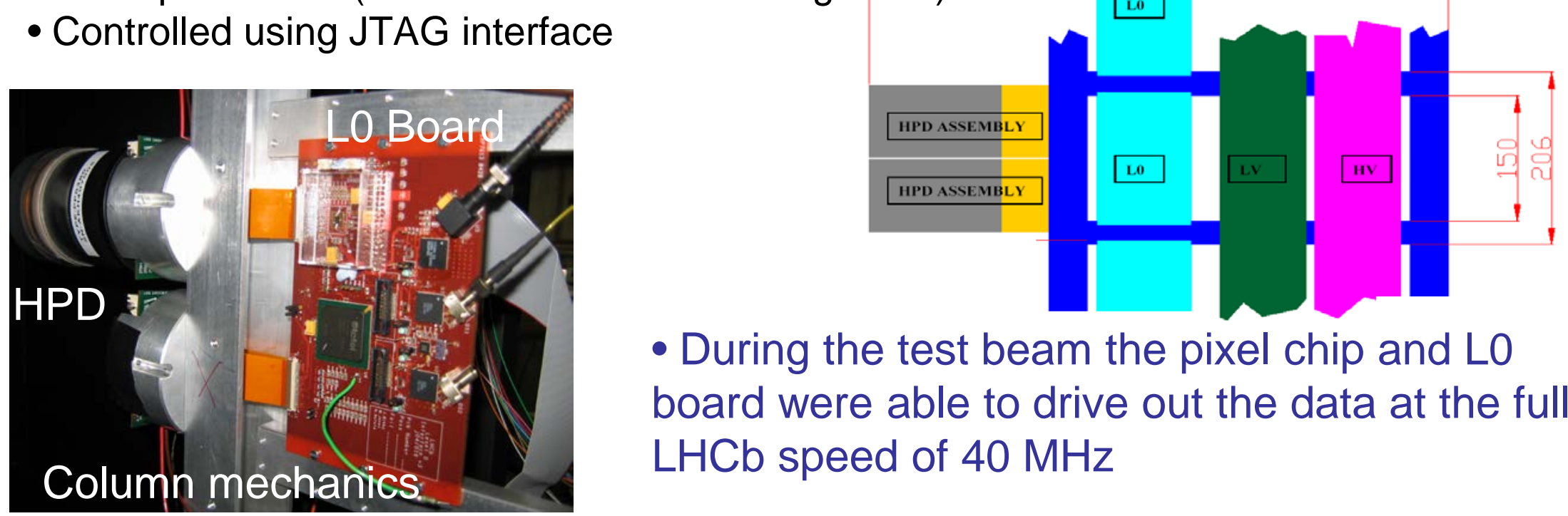

- During the test beam the pixel chip and LO board were able to drive out the data at the full $\mathrm{LHCb}$ speed of $40 \mathrm{MHz}$

- 1.6Gbit optical link also used successfully 


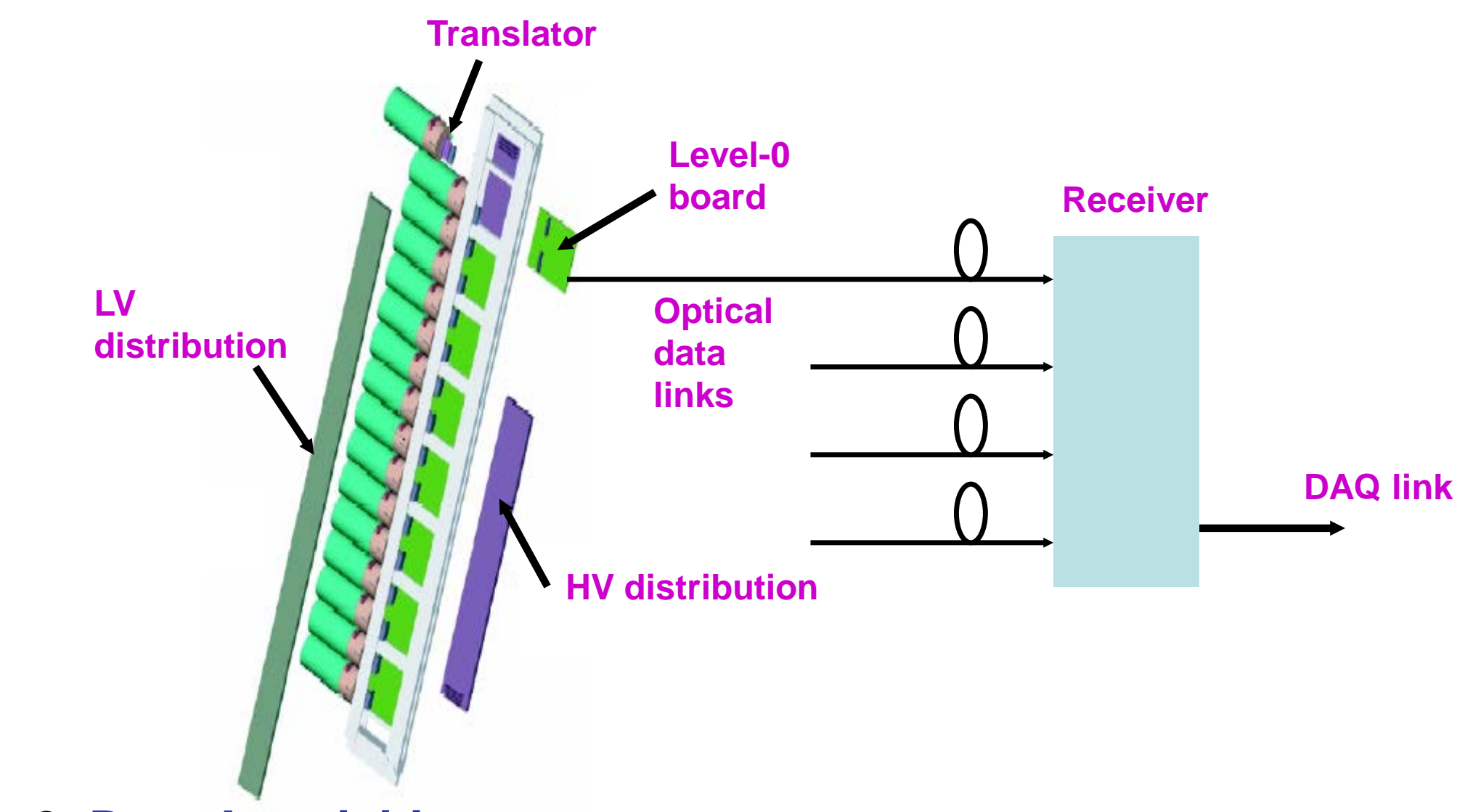

\section{Data Acquisition}

- Writes the LO data to disk

- Data from the L0 1.6 Gbit optical link is received by a custom receiver

- Triggered LO events were transferred to receiver card immediately, buffered there until the end of the PS spill

- Then copied to PC through PCl interface 


\section{Cherenkov Rings}
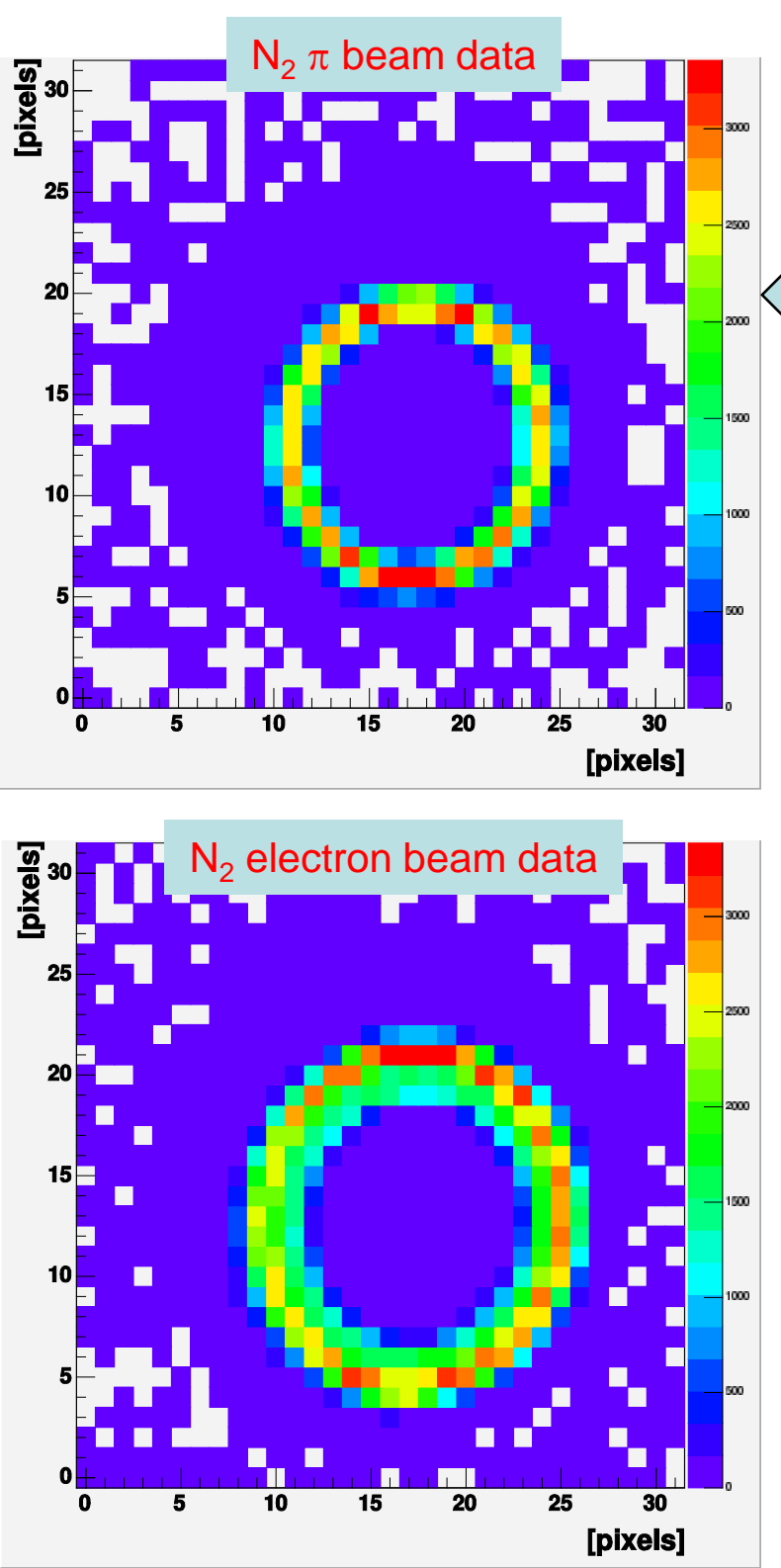

- Cherenkov rings observed from pion and electron beams with both :

- $\mathrm{N}_{2}$ radiator - rings on one HPD at a time Ring radii are in-line with expectations:

$$
\begin{array}{ll}
\pi & 13.9 \pm 0.2 \text { pixels } \\
\text { e } & 16.7 \pm 0.2 \text { pixels }
\end{array}
$$

(plots integrated over $\sim 30 \mathrm{k}$ events)

- $\mathrm{C}_{4} \mathrm{~F}_{10}$ radiator - rings spread on up to four HPDs (integrated plot shown)

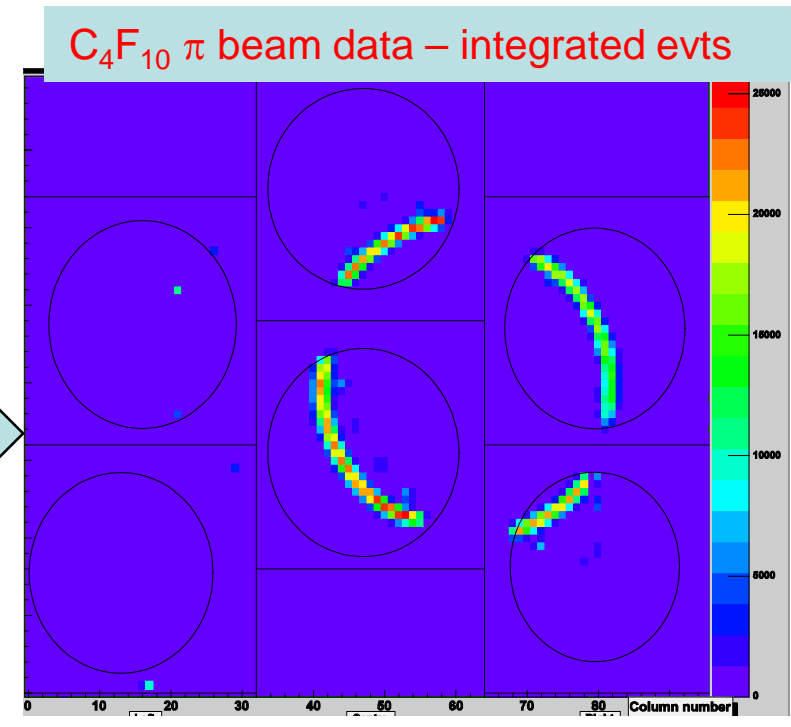

- As can be seen from plots, noise is at very low level $<0.1 \%$ 


\section{Calculating the p.e. yields}

- $\mathrm{N}_{2}$ data, $10 \mathrm{GeV}$ pion beam - count number of photoelectrons (p.e.) in rings

- Several effects change $N_{\text {p.e. }}$ distribution :

- Charge Sharing

(single p.e. $\rightarrow 2$ pixel hits)

$\rightarrow 2.5 \%$ effect

- Double hits

(two p.e. $\rightarrow 1$ pixel hit)

$\rightarrow 3 \%$ effect

- Asynchronous nature of set-up

- Beam trigger and chip clock not coincident (as they will be in LHCb) 500

$\rightarrow$ timing inefficiency

$\rightarrow$ additional efficiency factor $\sim 0.7$

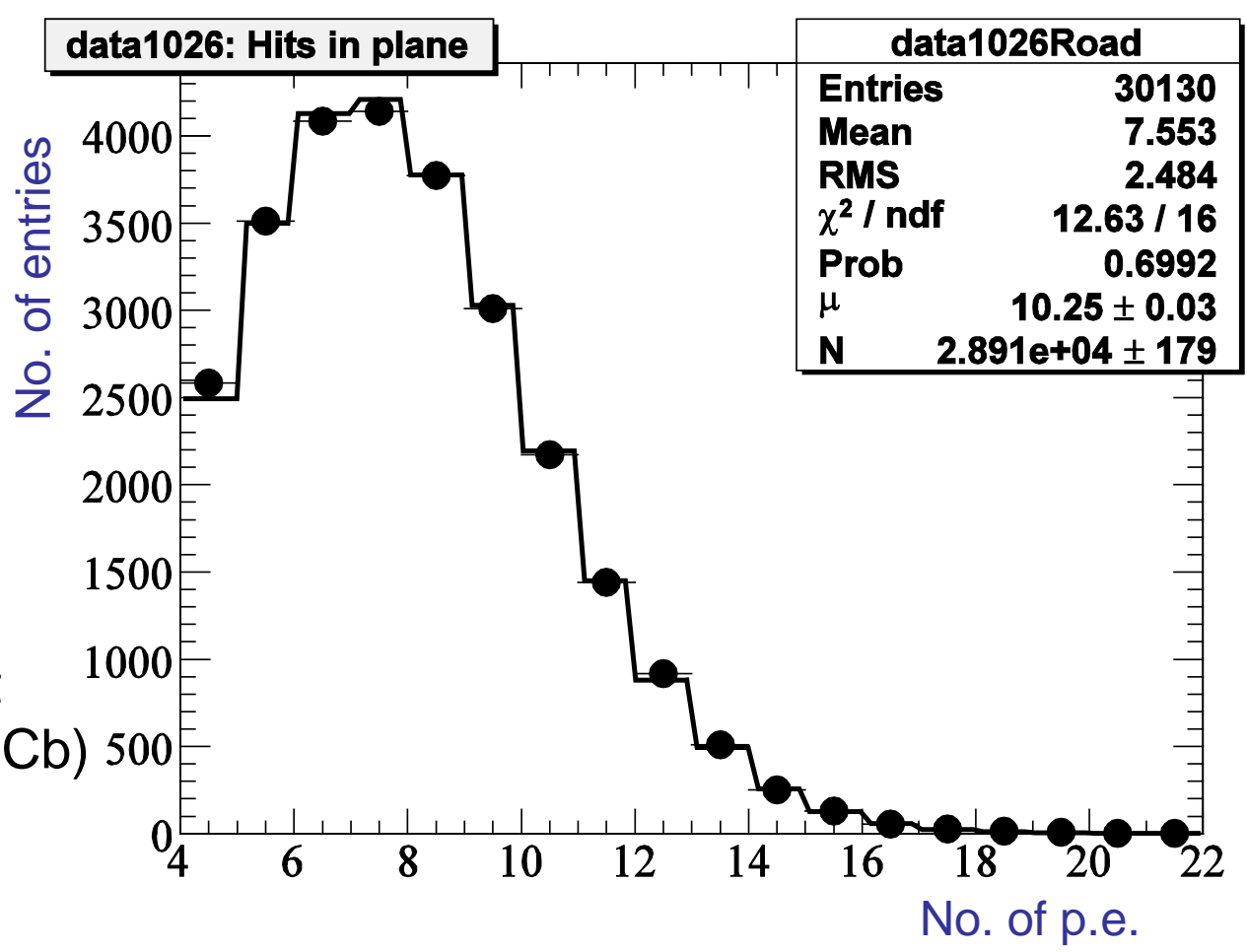

$\cdot$ Good fit to $N_{\text {p.e. }}$ with Poisson + these effects 
- Taking the detection efficiencies measured in lab ( $85 \%)$ find a good agreement between data and expectation :

\begin{tabular}{|c|c|c|c|}
\hline HPD & $\mu$ (fit) & $\mu$ (theory) & Ratio \\
\hline 1 & 7.8 & 6.0 & 1.3 \\
\hline 2 & 11.5 & 11.2 & 1.0 \\
\hline 3 & 8.8 & 8.9 & 1.0 \\
\hline 4 & 9.7 & 10.7 & 0.9 \\
\hline 5 & 10.1 & 10.0 & 1.0 \\
\hline 6 & 8.5 & 11.4 & 0.8 \\
4
\end{tabular} $\begin{aligned} & \text { Known timing problem } \\
& \text { with LO board } \\
& \text { Und Under investigation }\end{aligned}$

- Largest contribution to the error in $\mu$ (theory) from integration of measured QE of each tube, error at $10 \%$ level

- Detection efficiencies confirmed, performance as expected 


\section{Cherenkov Angle Resolution}

- $\quad \mathrm{N}_{2}$ data, $10 \mathrm{GeV}$ pion beam

- Width of Cherenkov angle distribution compared between DATA and GEANT4 based Monte Carlo :

- $\sigma_{\text {DATA }}=1.1 \mathrm{mrad}$

- $\sigma_{\mathrm{MC}}=1.0 \mathrm{mrad}$

- No. of effects reproduced in simulation :

\begin{tabular}{|l|l|}
\hline Chromatic & 0.4 \\
\hline Pixel & 0.8 \\
\hline Emission point & 0.3 \\
\hline Beam divergence & 0.2 \\
\hline Charge Sharing & 0.3 \\
\hline Total & 1.0 \\
\hline
\end{tabular}

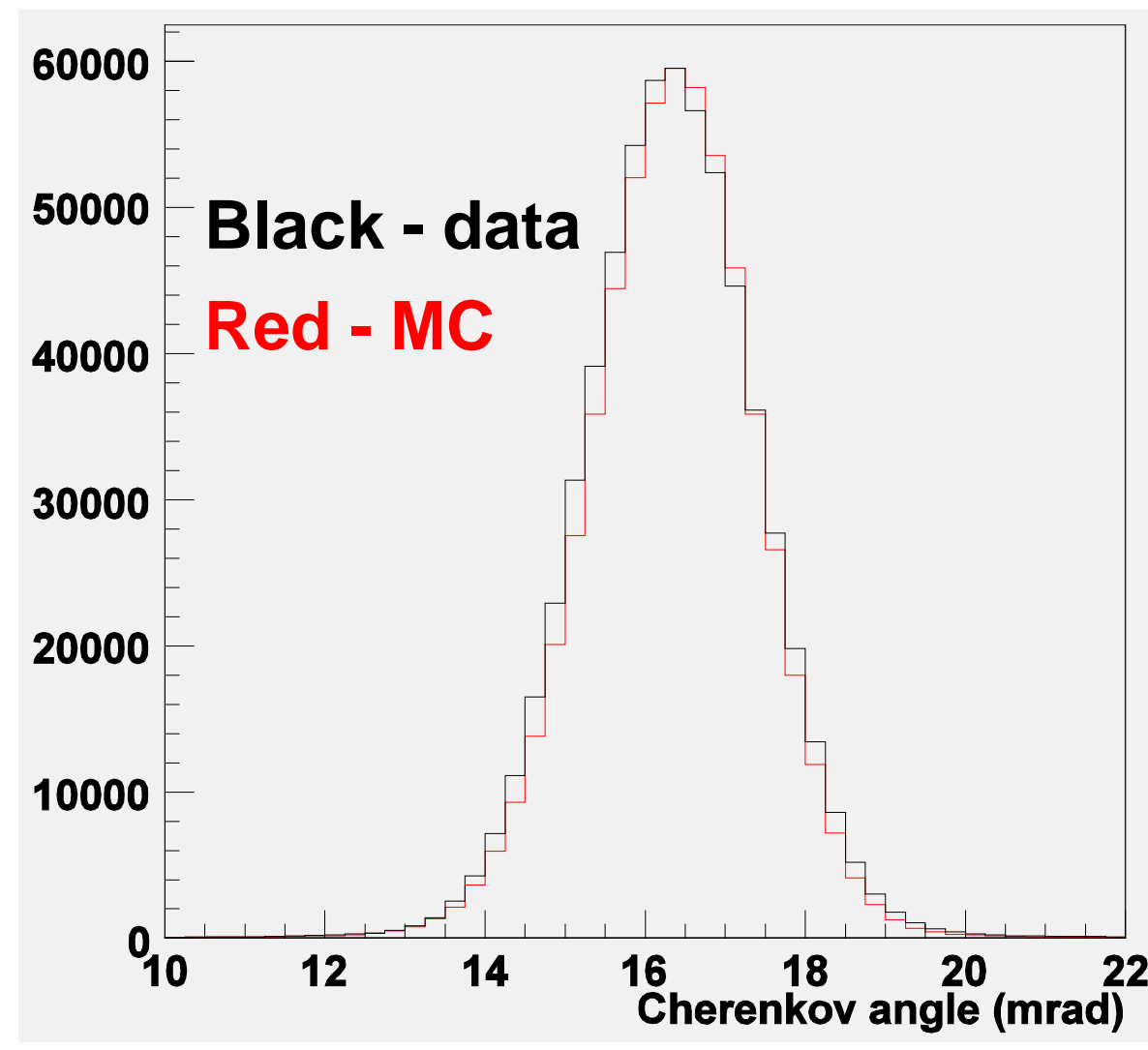

$\rightarrow$ in test-beam set-up are dominated by pixelisation

- Remaining difference under investigation 


\section{Conclusions}

- The first six pre-production HPD's tested in lab conditions

- Now also tested in beam conditions:

- Electron and pion Cherenkov rings observed- radii and photoelectron yields in line with expectations

- Successfully integrated pre-production HPD together with other $\mathrm{RICH}$ elements

- First meeting of readout electronics chain on-detector: binary chip and LO board together and operated at $40 \mathrm{MHz}, 1.6 \mathrm{Gbit}$ r/o through optical fibres

- Final mechanics and HPD Mounting scheme used successfully

- Pre-production HPD performance verified. Production of $\sim 550$ such devices now ongoing - now have 21 HPDs completed 


\section{Forming uncorrelated events}

- Size of charge sharing (single p.e. $\rightarrow 2$ pixel hits) and double hits (two p.e. $\rightarrow 1$ pixel hit) determined from the data

- Take an event in the data with $\mathrm{n}$ hits then form a new event with $\mathrm{n}$ hits taken from $n$ different events

- no contribution from charge sharing difference between no. of vertically and horizontally adjacent hits in the data and in the uncorrelated events $\rightarrow$ amount of charge sharing

- can count no. of times have two hits in same pixel $\rightarrow$ amount of double hits

- Confirm charge sharing result from runs taken with an LED light sourcevery low occupancy $\rightarrow$ can just count no. adjacent hits (limited statistics)
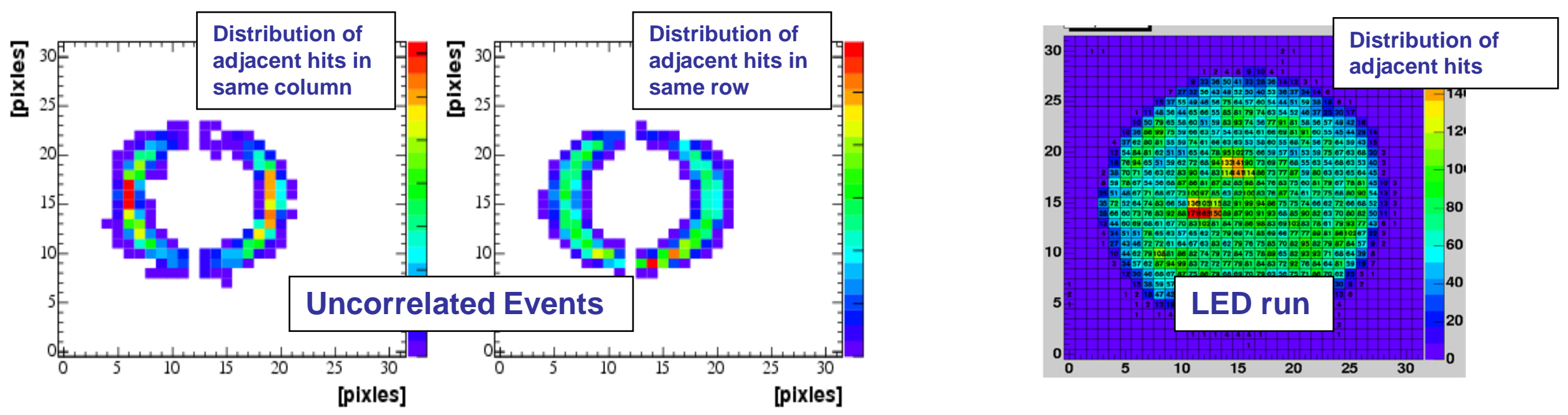


\section{Cooling Scheme}

- Complete column of HPDs will output 220W

- Use cooling plates attached to HPD aluminum column to remove this heat

- Thermal mat between copper skin and electronics components

- Cooling system: closed circuit circulation of water/ $\mathrm{C}_{6} \mathrm{~F}_{14}$

Aluminum honeycomb

Copper pipes

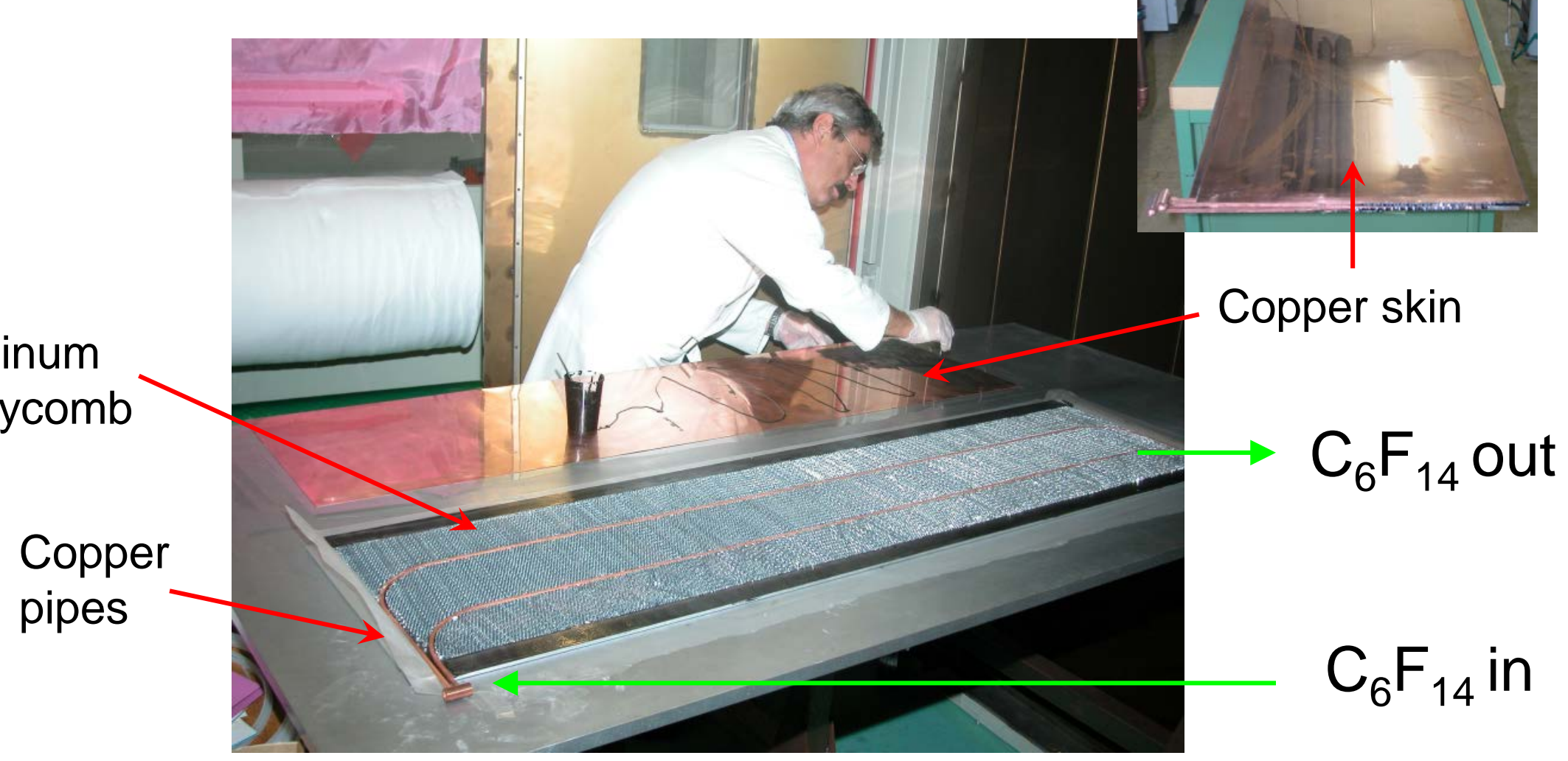



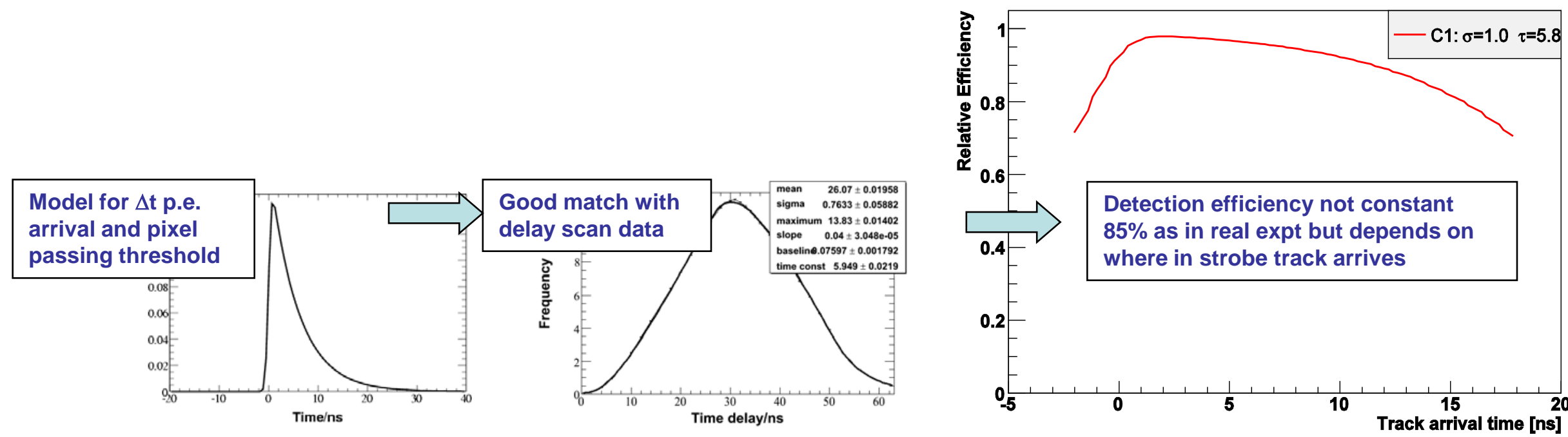